\title{
MMSE Acquisition of DSSS Acoustic Communications Signals
}

\author{
L. Freitag \\ Woods Hole Oceanographic Institution \\ Woods Hole, MA 02543 USA \\ lfreitag@whoi.edu \\ M. Stojanovic \\ Massachusetts Institute of Technology \\ Cambridge, MA 02139 USA \\ millitsa@mit.edu
}

\begin{abstract}
A method for acquisition of direct sequence spread spectrum (DSSS) signals based on a minimum mean-square error (MMSE) receiver is presented. An adaptive decision feedback equalizer (DFE), operating at the chip rate, uses the known spreading code as training data. A fixed number of un-modulated code periods is transmitted for acquisition purposes. An arbitrarily chosen initial code timing is hypothesized, and this timing is used to adapt the DFE and despread the chip estimates at its output. When the despreader output exceeds a threshold, lock is declared, and the receiver switches from acquisition to tracking mode. If the threshold is not exceeded during a prespecified amount of time, a different initial timing is hypothesized, and the receiver is re-initialized. The locally generated code can be advanced by a number of chips that is proportional to the length of the feedforward equalizer filter. The MMSE receiver also automatically acquires and tracks the Doppler shift that results from source-receiver motion using a phase-locked loop and integrated sample-rate interpolation. Code acquisition is demonstrated using experimental data with length 63 Kasami sequences transmitted at a chip rate of $10 \mathrm{kHz}$ for a resulting data rate of $\mathbf{1 5 0} \mathrm{bps}$. Up to 8 simultaneous users are reliably acquired and demodulated using the recursive least-squares (RLS) update algorithm.
\end{abstract}

\section{INTRODUCTION}

The code-division, multiple-access (CDMA) approach to multi-user communication has become very common in radio-frequency systems. While time-division, multipleaccess (TDMA) is also extremely common, and increasingly so as GSM mobile telephone systems expand, CDMA holds significant promise for underwater acoustic communications because it does not require precise time slot control. Timing control is difficult to establish reliably underwater because of the very slow speed of sound, while RF propagation delays in the atmosphere are by comparison almost insignificant, further motivating use of code-division. Two code-division methods are normally proposed for underwater spread-spectrum systems, frequency-hopping (FHSS) and direct-sequence (DSSS). While frequencyhopping offers the potential for simple receivers, it is unlikely to offer as effective use of bandwidth as phasecoherent signaling, thus providing incentive for research on viable DSSS receivers.

DSSS CDMA techniques are potentially viable for highcapacity multi-user systems and highly-spread, low-rate systems which aim to minimize the probability of detection by others. However, in contrast to terrestrial radio channels, the underwater acoustic channel offers little bandwidth (10 kHz rather than $1 \mathrm{MHz}$ typically), and significant intersymbol interference (ISI). Methods used in wireless mobile communications have traditionally been simple due to necessity, but attention has increasingly focused on more sophisticated direct-sequence receivers that include adaptive equalization. Techniques developed for RF systems that take into account the potential for extensive multipath are potentially applicable to the underwater channel, and the literature contains numerous examples of adaptive receivers for DSSS CDMA, for example [1].

The application motivating the work presented here is communication between multiple underwater systems, both fixed and mobile, operating in shallow $(200 \mathrm{~m})$ or very shallow $(15 \mathrm{~m})$ water and the surf-zone. The propagation of acoustic signals in this environment invariably includes multipath, typically with $5-25 \mathrm{msec}$ spread, but at close range in water depths of 100-200 $\mathrm{m}$ it may span 50-100 msec. Unlike deep-water propagation where the arrivals include only the direct path and a much-later bottom or surface bounce, shallow-water multipath often includes many rays of similar amplitude arriving close together. The interaction of acoustic signals with an energetic surface wave field further complicates propagation and increases time variability.

Previous work by the authors in phase-coherent, multiple-access receivers for underwater acoustic communication described in [2] and [3] focus on signal processing aspects of multipath equalization and interference suppression and assume that initial synchronization is already accomplished or done with side information. In the previous work a probe signal (FM sweep) was used to determine the starting time of a data packet. While the adaptive equalizer used in both [2] and [3] is similar, the spreading approaches are significantly different. In [2], a low-rate block code was used to provide coding gain against multi-access interference, while in [3] and [4], standard spreading codes were used. Timing acquisition suitable for the low-rate blockcode approach remains an open problem. In this paper, we combine the principles of adaptive chip-rate decisionfeedback equalization with a serial timing acquisition technique and present a solution for DSSS acquisition without side information. When combined with the hypothesis-test receiver of [3] a complete system may be realized.

The paper is organized as follows. Sec. II is a discussion of DSSS acquisition issues for underwater acoustic communications. Sec. III presents the system design and the algorithm for MMSE timing acquisition. Sec. IV is devoted to demonstrating the algorithm performance using in-water data. Finally, Sec. V summarizes the paper and outlines directions and issues to be addressed in future work.

\section{DSSS ACQUISITION}

The DSSS acquisition problem includes the estimation of signal parameters necessary to start the next phase of 
the receiver which may perform further refinement before switching to tracking mode. The signal parameters to be estimated are the delay $\tau$, and frequency shift $\omega$ due to Doppler, but the resolution requirements depend upon the type of receiver used after acquisition switches to tracking. While RF systems with very high chip rates (often in excess of $1 \mathrm{MHz}$ ) may search for the code offset $\tau$ using sophisticated serial search methods [6], [7], the relatively modest chip rates of acoustic systems (1-20 kHz) allow for use of a simple sliding correlator implemented as a matchedfilter in either the time or frequency domain. The length of the spreading code and the maximum expected Doppler shift controls the number of frequency shifts that must be searched and whether or not the replica code must be dilated or compressed in time to match the hypothesized Doppler.

For many underwater DSSS systems this approach is perfectly adequate. However, it requires that (1) the code length be longer than all of the channel delay spreads that will be encountered, and (2) the spreading gain is adequate to allow unambiguous detection of the desired signal under un-equal power situations or when multiple users are present. The unequal power situation is known as the nearfar problem, and it is particular difficult to solve in underwater networks because high-rate power control is nearly impossible due to the long propagation delay and because channel access is single duplex. Our system design goals include multipath channels and mobile underwater vehicles at arbitrary ranges and thus neither condition will be satisfied. Further, the adaptive equalizer proposed in [3] mitigates these effects to a large extent (in particular when multiple hydrophones are available), and so the acquisition system must have similar capability. It should also be noted that the receivers under consideration are all single-user receivers in that they do not require or have the capability to use information about other users. Multiuser receivers simultaneously decode data from all users and may sequentially decode and then remove the signals, beginning with the strongest. These techniques require channel estimation, making them difficult to use in dynamic multipath. The motivation for adaptive single-user receivers is discussed in additional detail in [1].

A number of adaptive methods for acquisition have been described in the literature. One uses an LMS adaptive filter combined with a serial search [8]. The adaptive filter operates in training mode, and the symbol-rate output of the despreader is used to generate an error signal that is used to update the filter. The mean-square error is used as an indication of convergence, and if it is low enough the LMS filter is examined to find the best time delay estimate. When the MSE does not satisfy the convegence criteria a new timing estimate is postulated and the process begins again. This approach is conceptually similar to that proposed in this paper, though the details of the receiver differ significantly.

Two other DSSS acquisition techniques also use MMSE criteria, [9] and [10]. In [9] short spreading sequences are combined with a parallel approach to estimation of symbol timing, while [10] uses a serial method and matches the filter length to the spreading sequence length, which must be fairly short if convergence is to be attained. As with [8], the filter is cross-correlated with the desired user's sequence to get the final estimate of time delay. As will be demonstrated later in this paper, in [10] the RLS algorithm was found to provide convergence superior to that of LMS.

\section{MMSE ACQUiSITION AlgORITHM}

The acquisition method proposed here is intended for applications where no side information is available, e.g. where bandwidth constraints are present or in LPI systems where the chip SNR is very low. The signal for the desired user includes a synchronization phase where a series of symbols that are not modulated with data are transmitted. The receiver must estimate the symbol timing during the synchronization phase. After acquisition the receiver switches to tracking and provides demodulated data to subsequent layers in the communication system that perform soft or harddecision error correction decoding and data framing. Thus the acquisition algorithm only needs to provide symbol synchronization within the number of un-modulated symbols alloted for timing recovery.

For a hypothesized initial timing estimate, the adaptive receiver is applied over an interval of time equal to a certain number of chips. If the timing estimate is close to the correct one, such that the useful signal is within the span if the filter, the adaptive equalizer will be able to converge. If not, erroneous operation will be observed through monitoring of the output of the de-speader, and a different sequence timing will be tested. An additional feature of the adaptive receiver is that it automatically performs phase synchronization. Integrated compensation of carrier shift as an integral part of the adaptive MMSE receiver eliminates the need for explicit phase synchronization that would have to be performed prior to acquisition in a conventional spread spectrum receiver.

While Kasami sequences of length 63 are used in the examples considered here, any short spreading sequence may be employed. The special properties of Kasami sequences are not exploited in the receiver, and Gold codes or randomly selected sequences may also be used with similar results.

The proposed acquisition process is shown in Fig. 1. The algorithm is based on the single or multi-channel decision feedback equalizer as described in [2] and [3]. The equalizer uses a number of un-modulated spreading sequences $\mathbf{c}$ as training data and after processing $N$ chips, where $N$ is the length of the code, the vector of soft chip decisions $\hat{\mathbf{d}}$ are de-spread. The value $\mathbf{c}^{\prime} \hat{\mathbf{d}}$ is then compared with a threshold used to establish whether or not lock as been achieved. If lock is established then the receiver switches from training mode to decision-directed mode where the modulated spreading sequence $\mathbf{c}$ is fed back to the equalizer with a one symbol delay ( $N$ chips). The delay has a small effect on tracking ability in a time-varying environment, but can be removed by operating two simultaneous equalizers using the method described in [3].

Four parameters govern the operation of the receiver: the length of the feedforward filter, the maximum number of code periods to train, the detection threshold used to establish lock, and the number of chips to advance when the receiver is not locked after the number of code periods to train has been exceeded. 


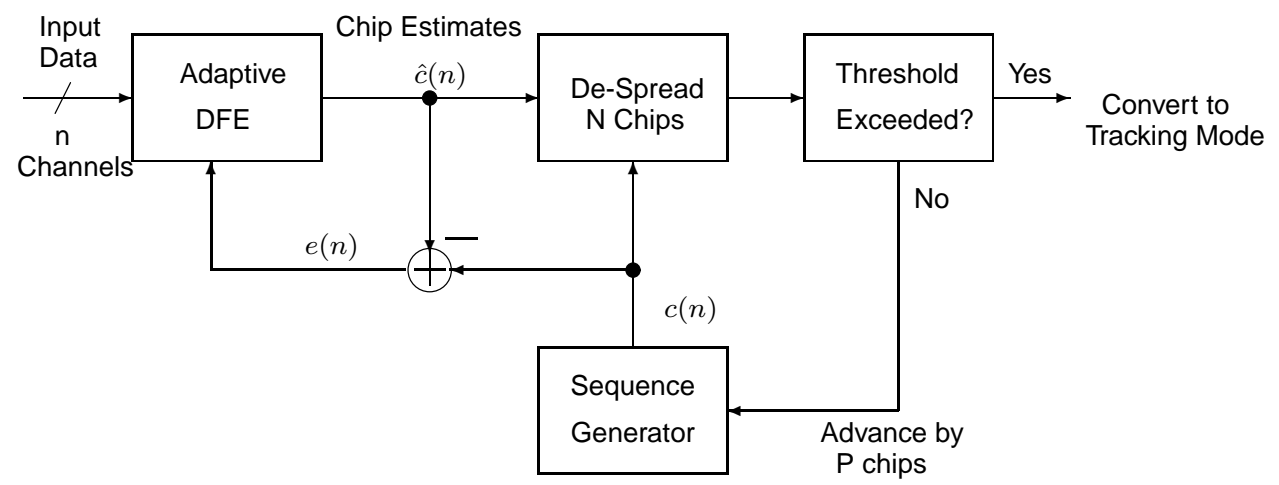

Fig. 1. MMSE Acquisition block diagram. The adaptive DFE may use any update algorithm, for example RLS or LMS, and also includes a 2nd order PLL.

When the code period is approximately of the same length as the feedforward filter, acquisition may be accomplished at a single initial timing estimate when the signal is present. Otherwise multiple timing hypotheses will be required, potentially increasing the acquisition time. The number of un-modulated symbols that are transmitted is related to the mean time to acquire. Unfortunately, the mean time to acquire is very difficult to estimate in a timevarying channel and a conservative estimate of the number of required symbols must be used. Faster acquisition can be accomplished by operating in parallel with multiple initial timing hypotheses. A further complication is the rate at which different adaptive filter algorithms used in the DFE converge. For example, the RLS algorithm converges quickly, but it is more computationally complex than algorithms from the LMS family. In this paper the adaptive step size version of the LMS algorithm is used [11].

For low-power systems operating on UUVs or in autonomous instruments, low complexity is preferred and a serial approach is proposed. As a design example, consider a 63 length spreading sequence, feedforward filter width of 40 fractionally-spaced taps (20 chips), and an equalizer convergence time of four spreading sequence lengths (252 chips). In this case, at most four initial chip timing hypotheses will be required in the absence of noise, and so the maximum time to acquire when the signal is present is 1000 chips. A typical shallow-water channel with a large array might require more symbols for convergence, perhaps doubling the the number of chips necessary for acquisition. At 5000 chips per second $0.2-0.5 \mathrm{~s}$ will be devoted to acquisition, which is reasonable if the packet length is on the order of five or more seconds, and at high spreading rates data transmissions may be much longer to be efficient.

Note that a DSP system that can support a DFE in realtime to receive data using a multi-channel array can be configured to operate the serial acquisition system in real-time as well. Thus there is no significant additional computational burden for acquisition since it uses the same signal processing elements, slightly re-configured for sequential timing acquisition. This is not the case for parallel acquisition, where the equalizer burden may be several times that required for real-time reception. For example, the case where 252 chips are alloted for acquisition at a given timing estimate and where the timing estimate is moved by 20 taps each time requires more than 10 times the computational capability of the receiver operating after lock is achieved.

\section{RESUlTS}

The acquisition method was testing using signals transmitted between two slowly drifting boats operating at short range (less than $1 \mathrm{~km})$ in shallow water $(20 \mathrm{~m})$. The chip rate is $10 \mathrm{kHz}$ and the carrier frequency is $25 \mathrm{kHz}$. The length 63 Kasami sequence was used for spreading, and 8 users were transmitted sequentially from one platform and received on a 4 element array with non-linear spacing $(0.3$, 0.6 and 0.9 m between elements 1-2, 2-3, 3-4 respectively). MMSE acquisition using a multi-channel DFE structure allows using all four hydrophones for both acquisition and detection. This can be a significant advantage, in particular when multipath creates extensive interference between adjacent symbols and multi-channel combining can reduce the ISI.

The data consists of short packets, each with 16 unmodulated sequences for use in acquisition, followed by 150 random data bits with spreading sequence applied. For initial capture and synchronization an FM sweep was transmitted prior to each packet. The FM sweep used $10 \mathrm{kHz}$ bandwidth and was $200 \mathrm{msec}$ long. The SNR is very high, but extensive multipath is present. A full view of the multi-

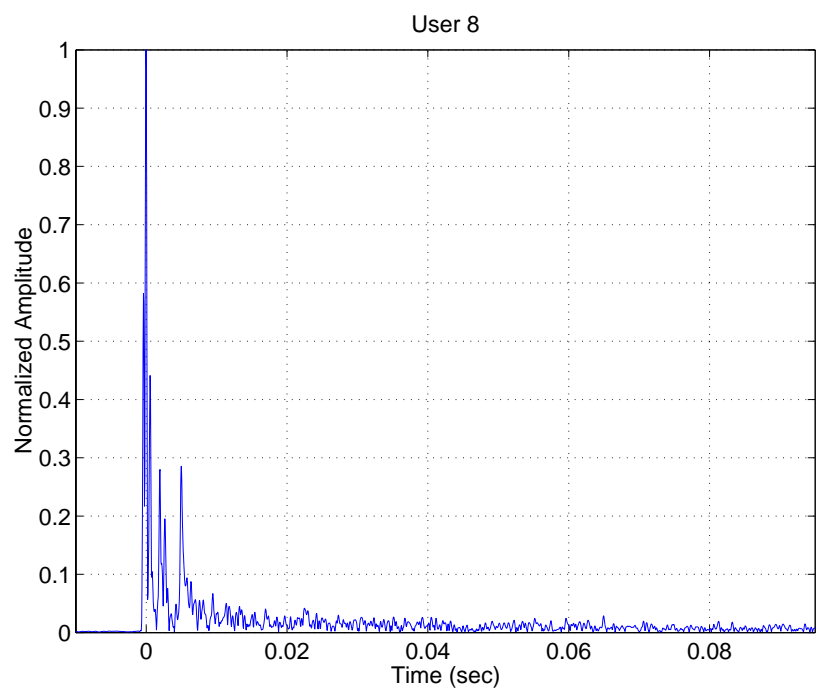

Fig. 2. Impulse response on one hydrophone channel for User 8. The rocky bottom and close range creates a very reverberant environment. 


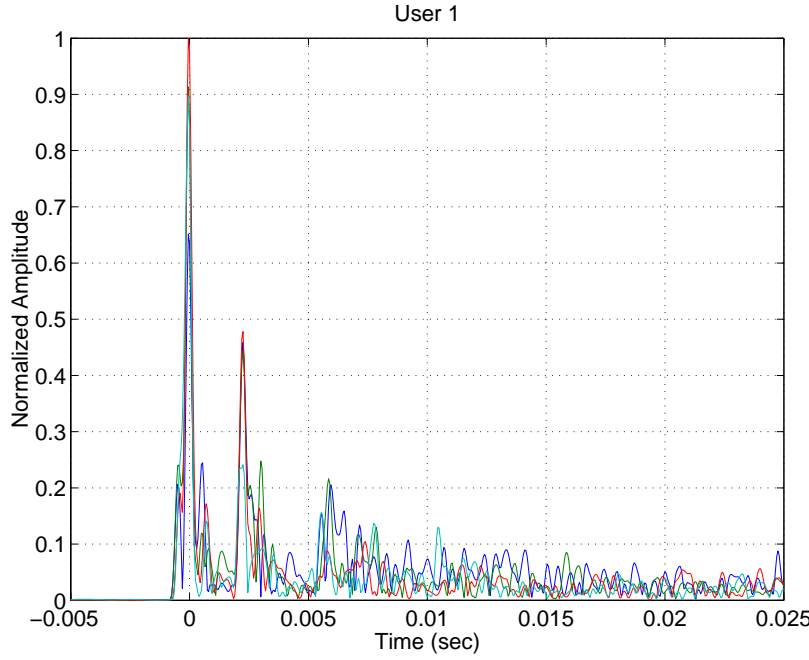

Fig. 3. Impulse response for User 1.

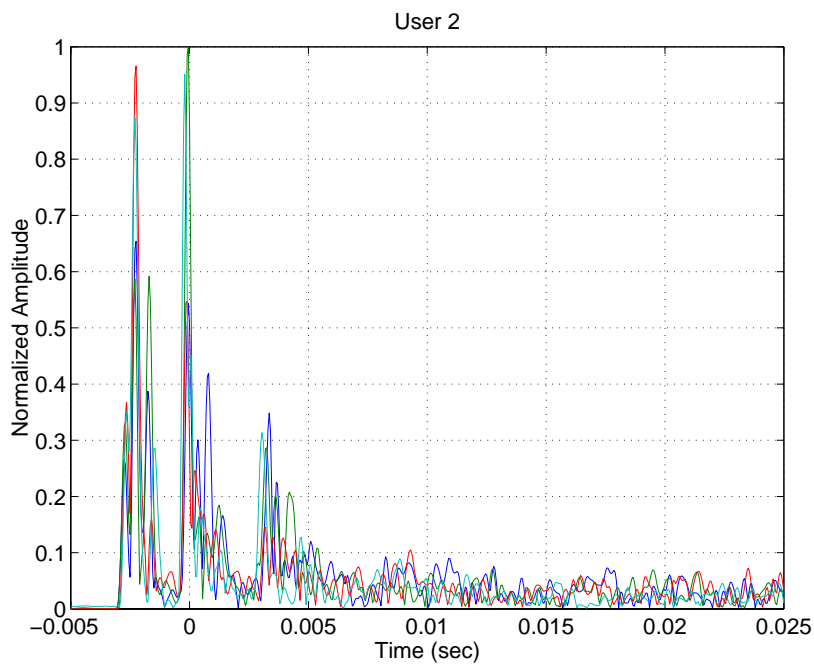

Fig. 4. Impulse response for User 2.

path is shown in Fig. 2, and a close-up of the multipath on all four channels for users 1 and 2 are shown in Figs. 3 and 4.

\section{A. Correlator Results}

The proposed MMSE method is intended for use in applications where conventional matched-filter approaches will not work. Thus it is first instructive to examine the output of a sliding correlator with just two users operating at approximately equal power when significant ISI is present. Fig. 5 shows the correlation of a two-user signal with their respective codes. The length of the spreading code is just 6.3 msec, and while the three major arrivals span approximately this same period, additional low-level arrivals extend much further in time. Thus the correlator output at equal power in Fig. 5 is not nearly as unambiguous as would be the case in an environment with less multipath.

As the power difference between two users grows, the ability of the correlator to unambiguously identify the desired user is reduced. For example, when user 2 is increased to $7 \mathrm{~dB}$ higher power than user 1 , the correlator for user 1 is not effective at all (Fig. 6). Thus, at best, the correlator approach will allow for two users of approximately equal
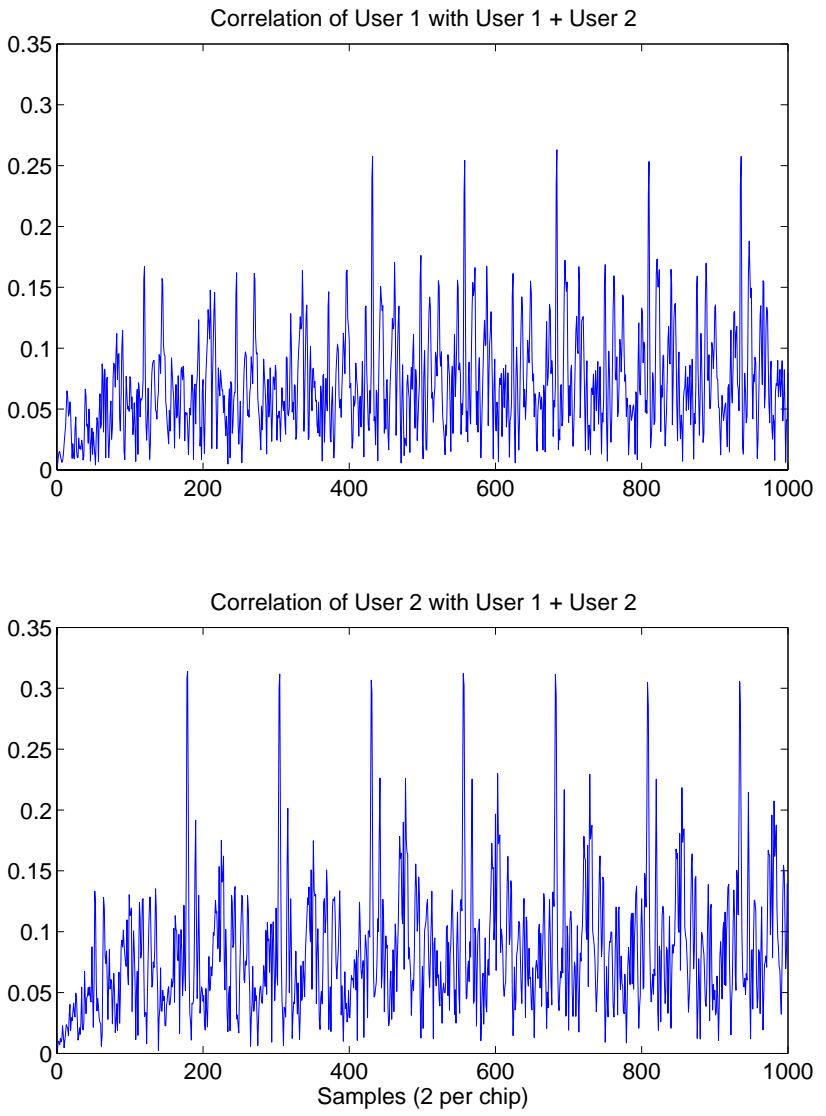

Fig. 5. Correlator outputs for user 1 (top) and 2 (bottom) when they both have near-equal power.

power. To support more users in this channel the length of the sequence must be lengthened so that the ISI will be resolved within the length of a spreading sequence. This has the undesired side effect of reducing data rate. Alternatively, a different method must be devised for acquisition in the presence of interfering users.

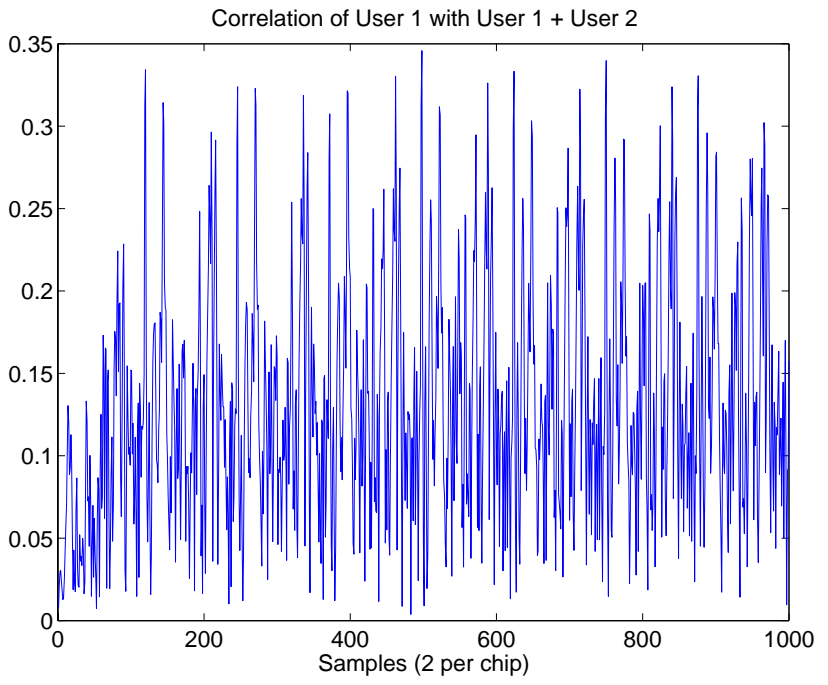

Fig. 6. Correlator output for user 1 when user 2 has $7 \mathrm{~dB}$ higher power.

\section{B. MMSE Acquisition and Decoding Results}

The use of the MMSE method is demonstrated using both the RLS and adaptive step-size LMS update algorithms. Two different scenarios are explored, two users with different power levels and multiple users (up to 8) with ap- 


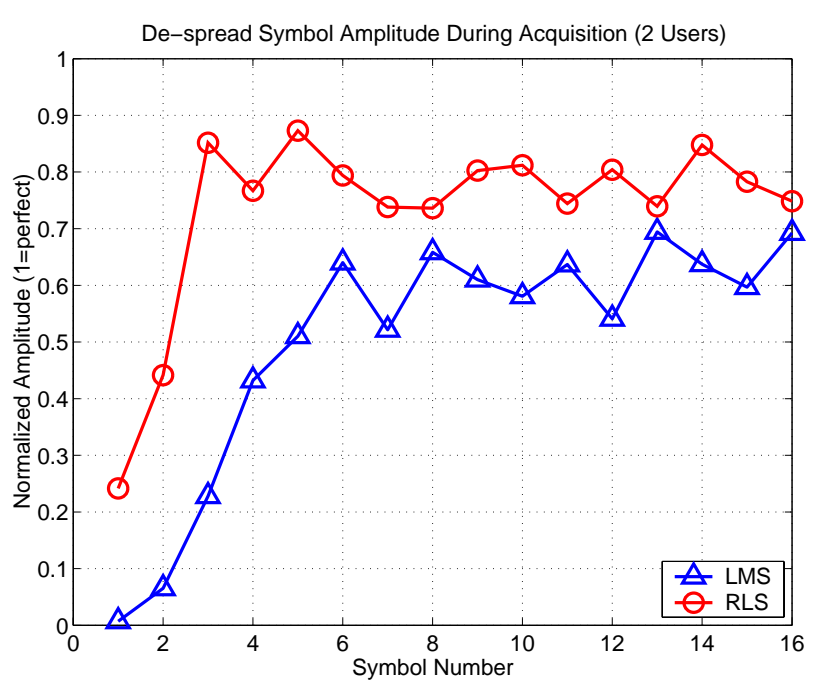

Fig. 7. Comparison of RLS and LMS algorithm convergence with 2 users.

proximately the same power level. In the tests done here the performance of the receiver after switching from acquisition to tracking mode is monitored to ensure that lock was successfully achieved and maintained throughout the data packet.

The tests are configured in all cases so that the desired user is always user 1. Signals from other users are added such that they are not chip synchronized and they are present prior to the start of the un-modulated data from user 1. The acquisition algorithm is initialized to start at, or just after, the desired user begins transmitting. The number of feedforward taps is 40 (20 symbols), and 10 feedback taps are used.

RLS versus LMS Acquisition. The advantages of RLS over LMS for fast training and tracking are well known [11] and have been demonstrated for the underwater acoustic environment as well [12]. In Fig. 7 the acquisition performance of each is compared on the same data set with two approximately equal-power users. The symbol amplitude after despreading is normalized such that perfect correlation is 1 . Use of a conservative acquisition threshold of 0.4 results in a time to acquire (essentially the time to train) for RLS that is half that required for LMS. While the performance difference between the two will vary with the number of filter taps, channel variability, and adaptation parameters, this result is typical. As postulated in the design example above, the number of symbols necessary to acquire is four when LMS is used, and just two for RLS from the point when the receiver encounters the start of the desired user's signal.

LMS Acquisition. The performance of the acquisition algorithm using LMS for equalizer adaptation at several different two-user power ratios is shown in Fig. 8. The time to when the output of the despreader exceeds the threshold varies from 3 to 5 periods. The LMS version does not perform nearly as well as RLS, but offers adequate two-user performance when their power levels are similar.

RLS versus LMS Demodulation. Just as RLS offers faster initial training, RLS tracks faster and offers better performance in a time-varying environment. This is demonstrated in a test where the same two users are combined and demodulated for user 1 with different power ratios to the

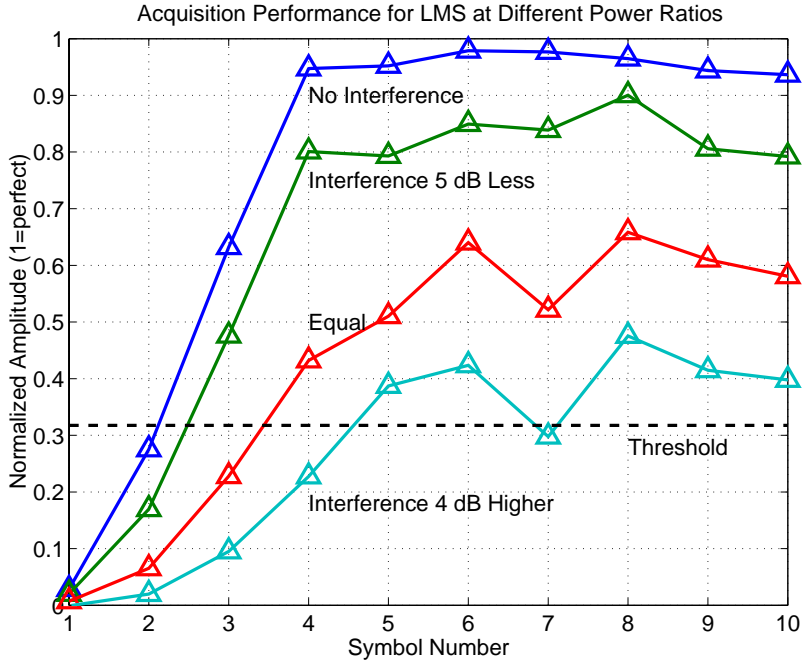

Fig. 8. LMS acquisition performance with 2 users at different power ratios.

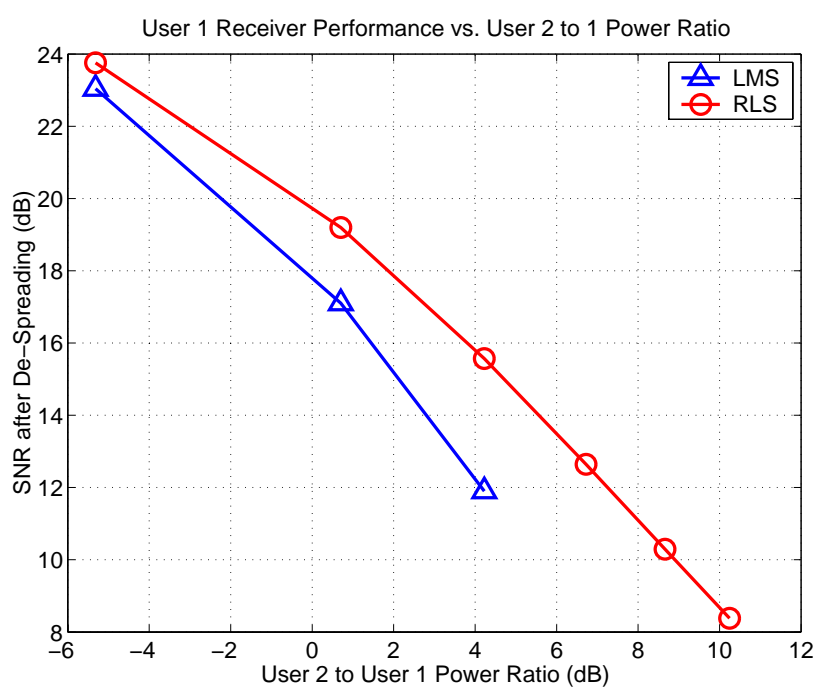

Fig. 9. Comparison of RLS and LMS algorithm performance with 2 users at different power ratios. Both receivers achieve greater than $22 \mathrm{~dB}$ output SNR when the desired user has $6 \mathrm{~dB}$ higher power than the interferer.

second user. In Fig. 9 the ratio of interferer to desired user is varied from $-6 \mathrm{~dB}$ (desired user has higher power), to 10 $\mathrm{dB}$ (interferer is significantly higher). The LMS receiver was unable to demodulate successfully in all cases, but the results show that at low interference ratios the performance difference is also low. However, when the second user's power is considerably higher RLS works when LMS will not.

RLS Performance with 3 to 8 Users. Successful acquisition and detection in the presence of up to 8 users ( 7 interfering) was demonstrated by summing all of the user signals together. The desired user (1) was acquired successfully and demodulated with 0 errors in all cases but one, where there was a single bit error. The signals are not normalized, simply added, so the output SNR is plotted with respect to the sum of the other users' power to show the linear relationship between input signal-to-interferer ratio and output SNR (at least in this case). 


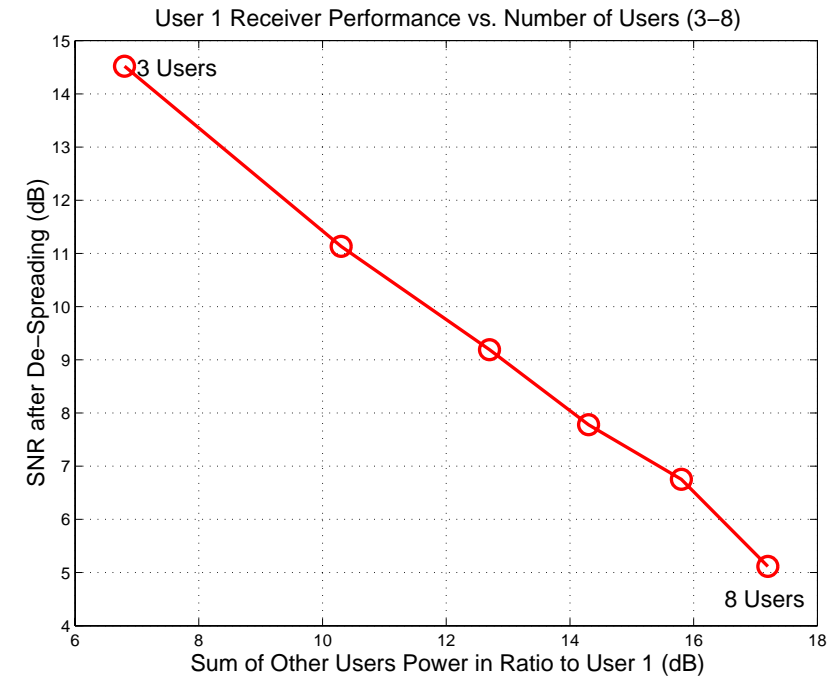

Fig. 10. Receiver performance on user 1 with 3 to 8 users total.

\section{CONCLUSION AND Future WORK}

The MMSE acquisition approach presented here is a conceptually simple way of acquiring un-modulated directsequence spread-spectrum signals. It provides all of the advantages of an adaptive DFE with integrated phase tracking, along with the complexity disadvantage inherent in that method. However, previous work has shown the advantage of adaptive processing [2] for multi-user spread-spectrum, and when this approach is used for decoding the data, the added sophistication of an adaptive acquisition approach has modest impact on receiver complexity.

As described here, the method is best suited for use with short spreading sequences as typically employed for multiuser communication. The use of short spreading sequences (for example, up to 255), allows the serial acquisition approach to be used efficiently, thus minimizing the computational complexity. In an application where low probability of intercept is required, the use of short-period spreading sequences is not desirable because they are easily identified by cyclostationarity detectors. Military DSSS systems often use very long-period spreading codes to ensure that the transmitted signal is as noise-like as possible. Fast timing acquisition of a long-period sequence is computationally difficult because a parallel approach must be used. The serial approach is possible, but it will be much slower because of the large number of possible timing estimates to be tested.

Future work includes simplification of the method to take advantage of symbol-rate processing when possible, and reduced-complexity combining at the front-end to provide initial multipath suppression prior to adaptive equalization. Fast acquisition of long-period codes remains a challenge with the system designer aided primarily by the fast decorrelation rate of the high-frequency acoustic channel which limits the required length of long-period codes.

\section{ACKNOWLEDGMENT}

This work was supported by the Office of Naval Research under contract number N00014-99-1-0287: Acoustic Communications for Very Shallow Water and the Surf-Zone.

\section{REFERENCES}

[1] M.Majmundar, N.Sandhu, and J.H.Reed, "Adaptive single-user receivers for direct-sequence spread-spectrum CDMA systems," IEEE Trans. Vehicular Tech., Vol. 49, No. 2, Mar. 2000. pp.379389.

[2] M.Stojanovic and L.Freitag, "Multiuser undersea acoustic communications in the presence of multipath propagation," in Proc. IEEE Oceans'01 Conference, Honolulu, HI, November 2001.

[3] M.Stojanovic and L.Freitag, "Hypothesis-feedback equalization for direct-sequence spread-spectrum underwater communications," in Proc. IEEE Oceans'00 Conference, Sept. 2000.

[4] M.Stojanovic and L.Freitag, "Acquisition of Direct Sequence Spread Spectrum Acoustic Communication Signals," in Proc. IEEE Oceans'03 Conference, San Diego, CA. Sept. 2003. pp. 279-286.

[5] L.Freitag, M.Stojanovic, S.Singh and M.Johnson, "Analysis of channel effects on direct-sequence and frequency-hopped spreadspectrum acoustic communication," IEEE Journal of Oceanic Engineering, vol.26, No.11, Oct. 2001, pp.586-593.

[6] J.K.Holmes, Coherent Spread Spectrum Systems, Malabar, FL: Krieger Publishing Company, 1990.

[7] J.G.Proakis, Digital Communications, New York: Mc-Graw Hill, 1995.

[8] M.G.El-Tarhuni and A.U.H.Sheikh, "Code acquisition of DS/SS signals in fading channels using an LMS adaptive filter," IEEE Trans. Commun., vol.2, No.4, Apr.1998, pp.85-88.

[9] U.Madhow, "MMSE interference suppression for timing acquisition and demodulation in direct-sequence CDMA systems," IEEE Trans. Commun., pp.1065-1075, Aug. 1998.

[10] R.Smith and S.L.Miller, "Acquisition performance of an adaptive receiver for DS-CDMA," IEEE Trans. Commun., vol.47, No.9, Sept. 1999, pp.1416-1424.

[11] S.Haykin, Adaptive Filter Theory, New Jersey: Prentice Hall 1986.

[12] L. Freitag, M. Johnson and M. Stojanovic, "Efficient equalizer update algorithms for acoustic communication channels of varying complexity," in Proc. Oceans'97, pp. 580-585, Oct. 1997. 